\title{
Pacemakers in a Reaction-Diffusion Mechanics System
}

\author{
R. H. Keldermann, ${ }^{1}$ M. P. Nash ${ }^{2}$, and A. V. Panfilov ${ }^{1}$ \\ Received January 31, 2006; accepted September 20, 2006 \\ Published Online March 23, 2007
}

\begin{abstract}
Non-linear waves of excitation are found in various biological, physical and chemical systems and are often accompanied by deformations of the medium. In this paper, we numerically study wave propagation in a deforming excitable medium using a twovariable reaction-diffusion system coupled with equations of continuum mechanics. We study the appearance and dynamics of different excitation patterns organized by pacemakers that occur in the medium as a result of deformation. We also study the interaction of several pacemakers with each other and the characteristics of pacemakers in the presence of heterogeneities in the medium. We found that mechanical deformation not only induces pacemakers, but also has a pronounced effect on spatial organization of various excitation patterns. We show how these effects are modulated by the size of the medium, the location of the initial stimulus, and the properties of the reactiondiffusion-mechanics feedback.
\end{abstract}

KEY WORDS: reaction-diffusion-systems, oscillations, modeling in biology, pattern formation, continuum mechanics, excitation-contraction-coupling, mechano-electrical feedback

\section{INTRODUCTION}

Reaction-diffusion (RD) equations are one of the most studied classes of partial differential equations (PDEs) in mathematics applied to biological, chemical and physiological sciences. ${ }^{(25)}$ They describe various processes of spatial organization in different systems with the most important solutions being non-linear waves, vortices and stationary Turing patterns. Each of these solutions have been

\footnotetext{
${ }^{1}$ Department of Theoretical Biology, Utrecht University, Padualaan 8, Utrecht $3584 \mathrm{CH}$, The Netherlands; e-mail: R.H.Keldermann@bio.uu.nl.

${ }^{2}$ Bioengineering Institute and Department of Engineering Science, The University of Auckland, New Zealand.
} 
studied extensively over the years using both analytical and numerical approaches. Non-linear waves can be found in many biological, physical and chemical systems. Examples of such waves include: chemical waves in the Belousov-Zhabotinsky (BZ) chemical reaction, ${ }^{(38)}$ and waves of carbon monoxide oxidation on platinum catalytic surfaces, ${ }^{(17)}$ both of which organize spatio-temporal patterns; and electrical waves in retinal and cortical nerve tissue, ${ }^{(12)}$ where they may underly neurological diseases such as epilepsy. ${ }^{(2,6)}$ Non-linear waves also control the morphogenesis of the Dictyostelium discoideum (Dd) amoeba ${ }^{(11)}$ and the initiation of the development of Xenopus oocytes after fertilization. ${ }^{(19)}$ One of the most practically important applications of non-linear waves are activation waves in the heart that give rise to cardiac muscle contraction.

The main function of the heart is to pump blood, which is achieved by the coordinated contraction of millions of cardiac cells (myocytes). This coordination is achieved via non-linear electrical waves that propagate through the cardiac tissue and initiate cardiac contraction. Problems in the electrical system of the heart may result in cardiac arrhythmias, e.g. rapid activation and spatial desynchronization of mechanical contractions in the heart, ${ }^{(26,30)}$ which cause the mechanical pump function of the heart to malfunction. In the US alone, more than 450,000 people die suddenly each year as a result of cardiac arrhythmias. ${ }^{(40)}$

Electrical excitation of a cardiac cell is a sudden change of its transmembrane potential, from its resting state (which is around $-90 \mathrm{mV}$ ), to an excited state (around $+10 \mathrm{mV}$ ). This rapid change of the membrane potential is a result of a complex interaction of different membrane ionic channels and pumps, which are time and voltage dependent. Excitation of a cardiac cell can be initiated by an electrical stimulus that produces an initial depolarization of the cell membrane from its resting state to exceed a certain threshold value (approximately $-60 \mathrm{mV}$ ). Once this threshold value is reached, a very rapid "all or none" response follows, and brings the membrane potential up to $+10 \mathrm{mV}$. This is called the depolarization phase. After depolarization, there is a repolarization phase during which the resting membrane potential is restored. However, during the repolarization phase cells are unable to respond to a subsequent stimulus, because not all the ionic channels have yet recovered from the depolarization phase. This is called the refractory period. In cardiac tissue, muscle cells are electrically coupled such that if one cell becomes excited (depolarized), the membrane potential of its neighboring cells also increases. If the threshold is reached in these cells, then they also become depolarized and in turn the membrane potentials of their neighbors is raised, and so on. This results in the formation of a propagating wave of excitation.

Normally, cardiac excitation is produced by a specialized group of cells in the heart (the sinus node) consisting of self-oscillating cells that periodically initiate propagating waves of excitation. The sinus node is a complex heterogeneous structure and the precise details of its function are not completely understood. 
Mathematical modeling is widely used to study wave propagation in the heart. There are many models available that describe the action potential of single cells. $(1,3,5,8,21,34)$ Important for these models is that they should cover the fundamental features, which we described above. In particular cells must be excitable, they require a refractory phase, and depolarization must propagate as a non-linear wave. $^{(13)}$

Usually, propagation of non-linear waves is accompanied by other important processes of which one of the most fundamental is mechanical deformation. Nonlinear waves during Dd morphogenesis induce cell-motion, which substantially affects wave dynamics. ${ }^{(36)}$ Waves in BZ reactions cause deformation of the gel, (37) which in turn affects spiral wave dynamics. ${ }^{(24)}$ In the heart, electrical waves initiate contraction of cardiac tissue.

The process by which cellular depolarization causes myocytes to contract is called excitation-contraction coupling (ECC). During depolarization, a small quantity of calcium ions flows into the cell through the so called L-type calcium channels, which are located on the cell membrane. This calcium current causes a subsequent release of a much larger quantity calcium stored within specialized cellular compartments called the sarcoplasmatic reticulum. The increase of the intracellular calcium concentration initiates cardiac contraction via several conformational changes of interacting proteins: troponin-C, actin, and myosin. ${ }^{(18)}$

Contraction of cardiac tissue also affects the process of wave propagation, (18) which is called mechano-electrical feedback (MEF). MEF may have both antiarrhythmic and arrhythmogenic consequences, and has been studied for well over a century. ${ }^{(18)}$ For example, mechanical deformation has been shown to alter the electrical properties of myocytes ${ }^{(31)}$ and play an important role during arrhythmias. ${ }^{(10)}$ Two phenomena that involve MEF are commotio cordis ${ }^{(20)}$ and the precordial thump. Commotio cordis is a situation in which a blunt, non-penetrating object strikes the chest and produces cardiac arrhythmias that may lead to sudden death. The precordial thump is a life saving technique during cardiac arrest in which the heart beat can be re-activated by delivering a sharp blow to the chest.

The precise mechanisms underpinning the role of MEF in arrhythmogenesis remain unclear. Modeling can be a helpful tool to investigate the underlying mechanisms of cardiac arrhythmias. Although the interplay of mechanical deformation with the dynamics of RD systems is an important phenomenon, most studies have separated mechanical deformation from non-linear wave propagation.

We have previously presented a general framework for studying the effects of mechanical deformation on RD systems. ${ }^{(27)}$ There we described a deformable, excitable medium capable of conducting non-linear waves of excitation. We did not aim to perform a detailed study that combines physiologically detailed excitation models with biophysically-based contraction models and realistic cardiac anatomy. Rather, we have started from a fundamental description of the electromechanical coupling using basic models of these processes together with simple rectangular 
geometries. This is because our aim was to study the fundamental effects that MEF may have on wave propagation, and thus elucidate the underlying mechanisms. The RD system was defined in a general curvilinear coordinate system, with a metric tensor determined by the equations of continuum mechanics. In turn, deformations were initiated and controlled by the RD system. We illustrated this concept of a coupled reaction-diffusion-mechanics (RDM) system using a simple two variable RD model of cardiac excitation. However, the model in ref. 27 lacks several important feedback mechanisms, including a representation of the stretch activated channels, which describe one of the main effects of MEF in cardiac tissue.

In a subsequent study, ${ }^{(29)}$ we introduced another RDM model, which contained a description of the stretch activated channels. There, we found that mechanical deformation can induce automatic pacemaking activity. Pacemaking was shown to occur after a single electrical or mechanical stimulus in an otherwise non-oscillatory medium. We showed that pacemaking activity resulted from stretch of the medium and subsequent depolarization via the stretch activated channels. However, we restricted our study to a small parameter region and only investigated the onset and behavior of a single pacemaker.

In this article we extend the results of our previous paper ${ }^{(29)}$ and study the effects of different conductivities for stretch activated channels, multiple pacemaker sites and parametric gradients on drift and excitation patterns.

\section{MODEL AND INTEGRATION METHODS}

The Reaction-Diffusion equations are given by the following expression:

$$
\partial \mathbf{V} / \partial t=\nabla \cdot(\mathbf{D} \nabla \mathbf{V})+\mathbf{F}(\mathbf{V})
$$

where $\mathbf{V}$ is a vector of concentrations, $\mathbf{D}$ is a diffusion tensor and $\mathbf{F}(\mathbf{V})$ is a nonlinear vector function. The number of components and properties of the diffusion tensor are different for different types of systems.

For cardiac tissue, the minimal number of components of $\mathbf{V}$ is two: one variable that describes the transmembrane potential and one variable that controls the recovery processes. ${ }^{(9)}$ Such low dimensional models can reproduce some important measurable characteristics of cardiac tissue, such as action potential restitution properties, the general shape of the action potential in the heart, and the effects of tissue anisotropy and heterogeneity. ${ }^{(1,33)}$ By adding one or two extra variables these models can also describe experimentally measured conduction velocity restitution and the exact shape of the action potential. ${ }^{(7)}$ However, low dimensional models do not describe detailed biophysical mechanisms of excitation that occur due to different dynamics of ionic channels on the cardiac membrane. To describe ionic channel dynamics one should use so-called ionic models for cardiac tissue that are based on the founding paper by Hodgkin and Huxley. ${ }^{(14)}$ 
Ionic models describe the properties of each individual ionic channel and are based on experimental studies of voltage and time dynamics using voltage clamp techniques. Recent ionic models for cardiac tissue include around $60-100$ variables to model many details of ionic channel dynamics identified in cardiac cells. ${ }^{(28)}$ Both low dimensional and ionic models can be used to study wave dynamics in cardiac tissue. Low dimensional models are used as tools for more general qualitative studies of possible new effects, while ionic models are used for detailed quantitative studies of specific effects for which molecular membrane mechanisms are established, e.g. drug applications, genetic disorders, etc.

\section{Modeling Elastic Deformations}

During a normal heart beat, cardiac cells deform up to the order of $15 \%$. ${ }^{(23)}$ Therefore, finite deformation elasticity theory must be applied to describe the deformations in the medium. Following standard continuum mechanics, we use two coordinate systems to describe the deformations. Assume that $\mathbf{x}=\left\{x_{i}\right\}$ describes the present (deformed) position in rectangular Cartesian coordinates of a material particle that occupied the location $\mathbf{X}=\left\{X_{M}\right\}$ in the reference (undeformed) configuration. The deformation gradient tensor, $\mathbf{F}$, transforms the undeformed line segment, $d \mathbf{X}$, into the deformed line segment, $d \mathbf{x}$, by $d \mathbf{x}=\mathbf{F} d \mathbf{X}$ with $F_{M}^{i}=\frac{\partial x_{i}}{\partial X_{M}}$. The right Cauchy-Green deformation tensor, $\mathbf{C}$, describes how each component of the undeformed line segment $d \mathbf{X}$ contributes to the squared length of the deformed line segment $d \mathbf{x}$ and is defined in terms of the deformation gradient tensor:

$$
\mathbf{C}=\mathbf{F}^{T} \mathbf{F} \quad \text { or } \quad C_{M N}=\left\{\frac{\partial x_{k}}{\partial X_{M}} \frac{\partial x_{k}}{\partial X_{N}}\right\}
$$

The right Cauchy-Green deformation tensor is independent of rigid body motion. We can define three principal components, which remain unchanged under coordinate rotations at a given state of deformation: $I_{1}=\operatorname{tr} \mathbf{C}, I_{2}=1 / 2\left[(\operatorname{tr} \mathbf{C})^{2}-\operatorname{tr} \mathbf{C}^{2}\right]$ and $I_{3}=\operatorname{det} \mathbf{C}$. Next, we introduce the Lagrangian Green's strain tensor, $\mathbf{E}$, which is defined by:

$$
\mathbf{E}=\frac{1}{2}(\mathbf{C}-\mathbf{I}) \quad \text { or } \quad E_{M N}=\frac{1}{2}\left(C_{M N}-I_{M N}\right)
$$

where $\mathbf{I}$ is the unitary tensor. Note that both $\mathbf{C}$ and $\mathbf{E}$ are symmetric tensors by definition. To represent material behavior independent of rigid body motion, we use the second Piola-Kirchhoff stress tensor, $T^{M N},{ }^{(22)}$ that represents the force per unit undeformed area, acting on an infinitesimal element of surface in the reference configuration.

The equations that govern finite deformation elasticity arise from the conservation of linear momentum following Newton's laws of motion ${ }^{(22)}$ and for static 
equilibrium are given by:

$$
\frac{\partial}{\partial X_{M}}\left(T^{M N} F_{N}^{j}\right)=0
$$

The relationship between the stress and strain $\left(T^{M N}\right.$, and $C_{M N}$ or $\left.E_{M N}\right)$ is given by an appropriate constitutive relation and is described later.

\section{Coupled Reaction-Diffusion-Mechanics Equations}

Our model is based on the concept of a deforming RD medium, which we introduced in ref. 27 . In order to mathematically couple the RD system and the mechanical equations, the relationship between Eqs. (1) and (4) must be considered. In general, each term in Eq. (1) may depend on the state of deformation, resulting in:

$$
\partial \mathbf{V} / \partial t=\nabla \cdot(\mathbf{D}(\mathbf{C}) \nabla \mathbf{V})+\mathbf{F}(\mathbf{V}, \mathbf{C})
$$

where $\mathbf{C}$ is the right Cauchy-Green deformation tensor, defined by Eq. (2).

The effects of the RD equations on the mechanics equations arise from the fact that some variables $V_{i}$ in Eq. (1) control the development of the active stress in the medium. For example, the contractile force developed by cardiac myocytes is determined by the intracellular concentration of calcium ions $\left[\mathrm{Ca}^{2+}\right]_{i}$, which is one of the variables of the RD model describing cardiac cells. ${ }^{(15)}$ In a modeling context, we split the second Piola-Kirchhoff tensor into active and passive stress components (see ref. 27), and use one or more of the variables of Eq. (5) to modulate the active stress development:

$$
T^{M N}=T_{p}^{M N}(\mathbf{C})+T_{a}^{M N}\left(\mathbf{C}, V_{a}\right)
$$

where $T_{p}{ }^{M N}(\mathbf{C})$ and $T_{a}{ }^{M N}\left(\mathbf{C}, V_{a}\right)$ represent the passive and the active tissue response, respectively. In ref. 27 , passive tissue properties were chosen to obey the isotropic Mooney-Rivlin constitutive law, and active tissue properties were also considered to be isotropic, and was determined using $T_{a}{ }^{M N}\left(\mathbf{C}, V_{a}\right)=V_{a} C^{M N}$. Other mechanical conditions, such as non-isotropic active stress, non-linear passive tissue properties, etc. can be implemented by modifying the terms in Eq. (6).

In this study, we used the following RDM model:

$$
\begin{aligned}
\frac{\partial u}{\partial t} & =\nabla^{2} u-k u(u-a)(u-1)-u v-I_{S} \\
\frac{\partial v}{\partial t} & =\epsilon(u)(k u-v) \\
\frac{\partial T_{a}}{\partial t} & =\epsilon(u)\left(k_{T} u-T_{a}\right)
\end{aligned}
$$




$$
\begin{aligned}
& \frac{\partial}{\partial X_{M}}\left(T^{M N} \frac{\partial x_{j}}{\partial X_{N}}\right)=0 \\
& T^{M N}=\frac{1}{2}\left(\frac{\partial W}{\partial E_{M N}}+\frac{\partial W}{\partial E_{N M}}\right)+T_{a} C_{M N}^{-1} \\
& \nabla^{2} u=\frac{\partial}{\partial X_{M}}\left(\sqrt{C} C_{M N}^{-1} \frac{\partial u}{\partial X_{N}}\right)
\end{aligned}
$$

To describe non-linear waves of cardiac excitation we use a low dimensional model based on the Aliev-Panfilov model. ${ }^{(1)}$ Here, $u$ is a dimensionless representation of the transmembrane potential and $v$ is a dimensionless variable that describes the recovery properties of the tissue. The term $(-k u(u-a)(u-1)-u v)$ represents the total transmembrane ionic current per unit area and controls the fast processes, such as the initiation and upstroke of the action potential. ${ }^{(27)}$ The threshold value $a$ represents the excitability of the tissue and is an important parameter for pacemaking ${ }^{(29)}$ ( $a=0.05$ unless otherwise noted). $k$ controls the magnitude of the transmembrane current ( $k=8$ in all simulations), and $\epsilon(u)$ determines the time scale of the recovery process and active stress: $\epsilon(u)=1$ for $u<0.05$, and $\epsilon(u)=0.1$ for $u \geq 0.05$. The other parameters do not have a clear physiological meaning, but are chosen in order to reproduce key characteristics of cardiac tissue, such as the shape of the action potential, refractoriness and restitution of action potential duration. $I_{S}$ represents the stretch activated current, which is described below.

The Aliev-Panfilov model ${ }^{(1)}$ is a low dimensional model for cardiac tissue that qualitatively describes the process of excitation and recovery of cardiac cells. However, the model does provide an experimentally based description of the restitution properties of action potential duration, which is important for the stability of wave propagation in the heart. The model presented in Eqs. (7)-(12) reproduces the shape of the action potential and the phenomenon of refractoriness as well as the effects of the stretch activated current $I_{s}$, which is described below. The values of the parameters of the model were found by fitting the overall characteristics of cardiac propagation. Although the Aliev-Panfilov model uses dimensionless units, simulation results can be compared to dimensional observations from experimental studies by comparing specific (dimensionless) model characteristics with experimental observations.

Deformation is modulated by the variable $T_{a}$ (described by Eq. (9)), which represents the active stress generated by the medium. The rate of tension development is determined by $k_{T}$ ( $k_{T}=10$ for all simulations).

The mechanical part of this model is unchanged from ref. 29. The active stress component of the second Piola-Kirchhoff stress tensor $T^{M N}$ in Eq. (11), is $T_{a} C_{M N}^{-1}$, and the passive elastic stress component, is expressed in terms of the derivatives of the strain energy function $(W)$ with respect to components of the Green's strain 
tensor from Eq. (3). The strain energy function was chosen to obey the MooneyRivlin constitutive law ${ }^{(16)}: W=c_{1}\left(I_{1}-3\right)+c_{2}\left(I_{2}-3\right)$, where $I_{1}$ and $I_{2}$ are the first two principal invariants of $C_{M N}$, and $c_{1}$ and $c_{2}$ are stiffness coefficients, which together with the parameter $k_{T}$ from Eq. (9) modulate the local deformations during contraction $\left(c_{1}=2, c_{2}=6\right.$ for all simulations, chosen to give rise to relative local deformations of approximately $15 \%$ following excitation). Due to motion of the material coordinate system, we used a general curvilinear expression given by Eq. (12) to evaluate the Laplacian in Eq. (7), with $C=\operatorname{det}\left(C_{M N}\right)$, which provides a diffusive membrane current per unit undeformed area.

The direct influence of contraction on excitation is given by the stretchactivated current $I_{s}$, known to be present in cardiac tissue. ${ }^{(18)}$ As in ref. 29 we use a generic description of the stretch-activated current into the model:

$$
I_{s}=G_{s}(\sqrt{C}-1)\left(u-E_{s}\right),
$$

where $G_{s}$ and $E_{s}$ are the maximal conductance and reversal potential of the stretch-activated channels, respectively. The stretch activated current in Eq. (13) is only present during stretch (i.e. when $\sqrt{C}>1$ ). The value of the parameter $E_{S}$ in our model was typically 1 , and describes the depolarizing effect of the current observed experimentally. ${ }^{(18,35)} G_{S}$ determines the outcome of the simulations with respect to drift and excitation patterns ( $G_{s}=0.6$ unless otherwise noted).

\section{Numerical Integration Methods}

The coupled electro-mechanical model was solved using a hybrid approach that combines an explicit Euler time integration scheme to compute RD equations of the medium, with non-linear finite element techniques to determine the large deformation mechanics of the tissue. To formulate the finite element integral equations, we introduced a weighting field of virtual displacements, $\delta \mathbf{v}=\left\{\delta v_{j}\right\}$, and the weak form of the stress equilibrium (Eq. (4)) is given by:

$$
\int_{V_{0}} T^{M N} F_{N}^{j} \frac{\partial \delta v_{j}}{\partial X_{M}} d V_{0}=\int_{S_{2}} \mathbf{s} \cdot \delta \mathbf{v} d S
$$

where $V_{0}$ is the undeformed volume and $S_{2}$ is the portion of the boundary subject to external tractions s. Eq. (14) was solved using the finite element method described in ref. 27.

The solution procedure is as follows: after $N_{\text {mech }}$ time integration steps for the RD equations (Eqs. (7)-(9)), the equations governing tissue mechanics are solved, using active stress components produced by the variable $V_{a}$ of the RD equations (Eq. (9)). Non-linear Newton iterations are performed to solve the stress equilibrium equations (Eq. (14)) and provide updated values of the deformation tensor C, which modulates excitation properties (via Eqs. (12) and (13)) for the subsequent $N_{\text {mech }}$ excitation time-steps. 


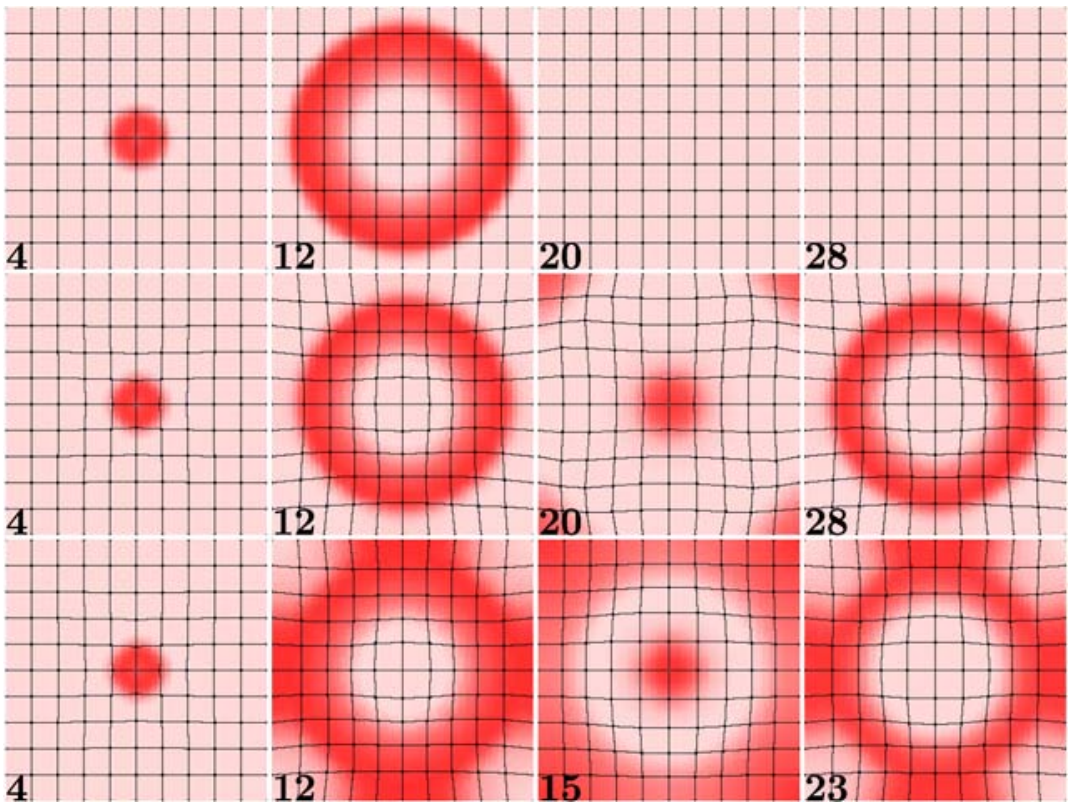

Fig. 1. Excitation patterns after application of a single stimulus at the center of the medium in Eqs. (7)(12). The upper panels show wave propagation in the absence of mechanical deformation. The middle panels show wave propagation in the presence of mechanical deformation with $G_{s}=0.2$. In the lower panels, $G_{s}=0.6$. The size of the medium is $61 \times 61$ grid points and $a=0.05$ for all simulations. Dark shadings represent regions where $u>0.6$. The period of the pacemakers in the middle and lower panels were 15.42 and 9.3 [t.u.], respectively. Times in time units [t.u.] are indicated at the lower left of each panel.

The model solution parameters were the following: Euler computations were performed using a time integration step of $\Delta t=0.03$ (dimensionless time units) and a space integration step of $\Delta x=\Delta y=0.6$ (dimensionless space units), consistent with previous studies. ${ }^{(29)}$ The mechanics mesh was defined using up to $16 \times 16$ finite elements. Each mechanical element contained $7 \times 7$ electrical grid points, and the value of $N_{\text {mech }}=3$ was used. Thus, the finite difference mesh was up to $97 \times 97$ grid points. No-flux boundary conditions were imposed for Eq. (7), and the boundaries of the medium were fixed in space for Eq. (14). Mechanically, the fixed boundaries are consistent with an isometric contraction regime.

\section{RESULTS}

\section{Single Pacemaker}

In Fig. 1, we illustrate the development of various patterns due to pacemaking in a deforming medium. The wave was initiated at the center of the medium at 


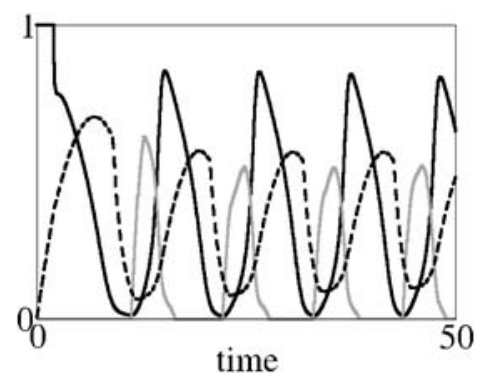

Fig. 2. Time course of the excitation variable $u$ (black line), active stress $T_{a}$ (divided by 5 ; dashed line) and stretch activated current (multiplied by 5; grey line) at the center of the medium for the computation presented in the lower panels of Fig. 1.

$t=0$. The upper panels show the process of wave propagation after one single stimulus in the absence of mechanical deformations. We see that the wave disappeared after propagating across the medium, after which the medium returned to a spatially homogeneous steady state configuration. Thus, in the absence of mechanical activity the reaction-diffusion model did not show oscillatory activity. The middle and lower panels of Fig. 1 illustrate the results obtained from the same initial stimulus, but in the presence of mechanical deformations. The middle panels show wave propagation from the same initial conditions for $G_{s}=0.2$. We see that in this case the mechanical deformation generates a pacemaker at the center of the medium, similar to that reported in ref. 29. Comparing the upper and middle panels at $t=12$, we observed that the conduction velocity of the propagating wave was slightly slower in the presence of mechanical deformation. This is because contraction initiates stretch in front of the wave (see also Fig. 2), thus the effective propagation distance is slightly increased. In the lower panels, we show a similar simulation with a higher value of $G_{s}=0.6$. We also observed the onset of a pacemaker, but the spatial activation pattern was altered: as the wave propagated away from the center, stretch was generated near the boundaries, which caused local activations in these regions, resulting in a diamond like pattern of excitation (see $t=12, t=23$, lower panels).

The mechanism of pacemaking is explained in Fig. 2, which shows the excitation variable $u$ (black line), active stress $T_{a}$ (dashed line) and the stretch activated current (grey line) for a point in the center of the medium.

Shortly after electrical activation, active stress $T_{a}$ was generated in the center causing contraction/shortening of the tissue. Because $I_{S}$ is only activated when tissue is elongated, no stretch activated current $I_{s}$ is generated during contraction (the grey line coincides with the $x$-axis). However, when the excitation wave propagated to some distance from the center, the contraction at the back of this wave caused stretch at the center, which led to an $I_{S}$ current in accordance with 


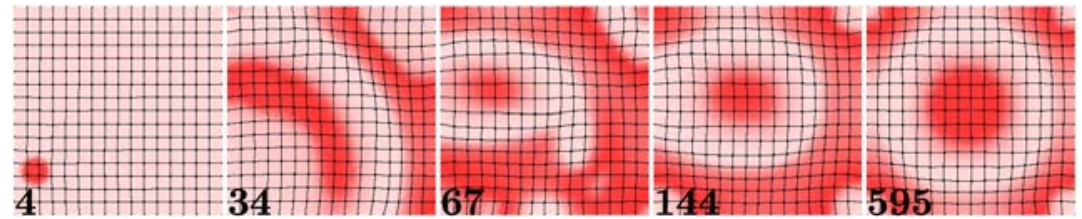

Fig. 3. Drift of a pacemaker starting at the point located in the lower-left corner. The size of the medium is $97 \times 97$ grid points, $G_{s}=0.6$ and $a=0.05$.

Eq. (13). As a result of this inward current, the tissue depolarized and generated a new action potential, which resulted in subsequent excitations. Note, that because the stretch of the tissue occurs after the contraction this results in a substantial delay between the activation wave and $I_{s}$, and that the tissue at that point recovers from the refractory period and is capable for new excitations. Therefore, mechanical deformation acting via the stretch activated current can initiate self-oscillatory activity in a RDM system. The activation patterns depend on the strength of the stretch activated channel, which is modulated by the value of $G_{s}$. In ref. 29, we showed that other parameters that increase $I_{s}$, or increase the excitability of the tissue, cause the onset of oscillations.

\section{Drift of a Single Pacemaker}

In Fig. 1 the initial stimulus was in the center of the medium. We have shown that if the initial excitation is not located in the center, then the pacemaker will drift, and for a large size medium it will approach the center. ${ }^{(29)}$ We show the results of such a simulation in Fig. 3. Here, the primary point of excitation is located at the lower left corner of the medium. We see that the pacemaker drifts to the center, however, this occurs in a few stages. We observe that the site of the second activation was substantially shifted from the site of the initial activation and that this second activation occurred in a large arc-shaped area (see $t=34$ ). The subsequent activation was again located closer to the center and occurred in a smaller area (see $t=67$ ). Note, however, that the overall excitation pattern still remained complex. Following the next excitation, at $t=100$, we observed a regular 'target' pattern type of excitations similar to that at $t=144$. Finally, the pacemaker drifted to the center of the medium where it stabilized (see $t=595$ ).

We have also performed a similar simulation with a decreased value of $G_{s}=0.5$. We obtained excitation patterns that were qualitatively similar to that shown in Fig. 3. We also observed an arc shaped area, which in turn was reduced to a point. Similarly, the point drifted to the center of the medium where it stabilized. To study the convergence of pacemaker drift, we determined the distance from the subsequent locations of the pacemakers to the center of the tissue. We show this for both $G_{s}=0.5$ (black) and $G_{s}=0.6$ (grey) on a logarithmic scale vs time in 


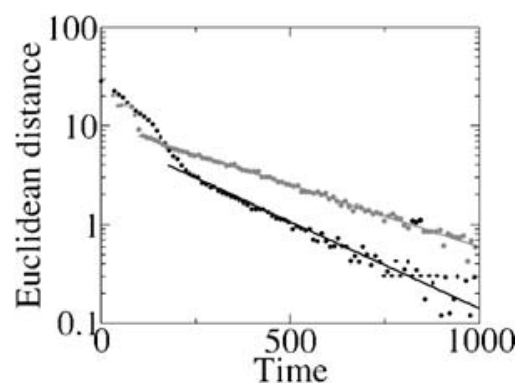

Fig. 4. Distance ([s.u.]) from the pacemaker site to the center of the tissue as a function of time in [t.u.] for $G_{s}=0.5$ (black line) and $G_{s}=0.6$ (grey line).

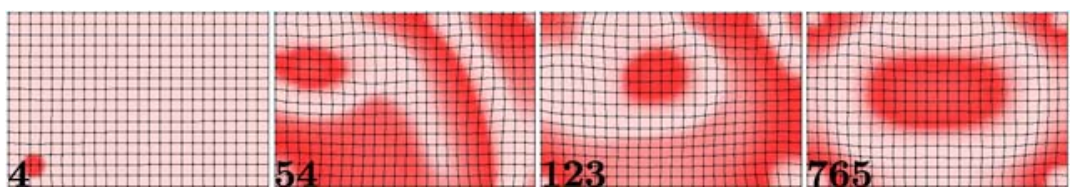

Fig. 5. Drift of a pacemaker starting at the point located in the lower-left corner. The size of the medium is $145 \times 97$ grid points, $G_{s}=0.6$ and $a=0.05$.

Fig. 4. The last part of the curves can be well fitted to a straight line, indicating exponential convergence of pacemaking activity to the center. Furthermore, we see that for $G_{s}=0.5$ the dependency has a steeper slope than $G_{s}=0.6$, and drifts faster to the center of the medium.

In the above simulations we used a square domain to investigate pacemaker drift. We also studied pacemaker drift in a rectangular domain with the horizontal side being 1.5 times longer than the vertical side (see Fig. 5). The primary point of excitation here is located at the lower left corner of the medium. We observed that the pacemaker drifted toward the center and that excitation patterns are qualitatively similar to that shown in Fig. 3: drift occurred in few stages, with fast initial drift and slower secondary drift. We also observed arc shaped areas during drift. When drift of the pacemaker stabilized in the center of the tissue, we see that the shape of the wave is elliptical, while this is circular in Fig. 3. This is a result of anisotropic stretch distribution in the horizontal and vertical directions for this domain shape.

\section{Drift of Two Pacemakers}

We also studied the dynamics of two coexisting pacemakers and their interaction (Fig. 6). The initial starting points were located symmetrically about the vertical mid-line at the left and the right boundaries (see $t=6$ ). We observed that both 


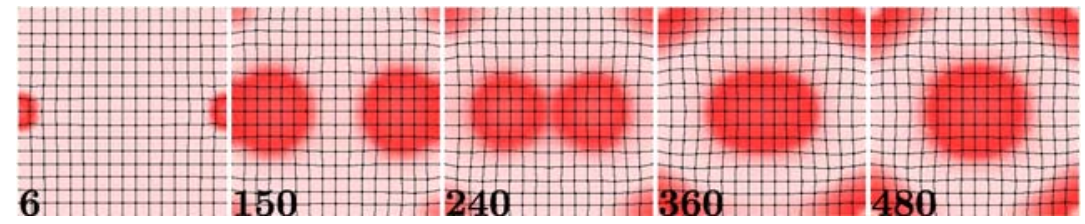

Fig. 6. Drift of two simultaneously initiated pacemakers located symmetrically about the vertical mid-line. The size of the medium was $97 \times 97$ grid points. Other parameter values were the same as those for Fig. 3.

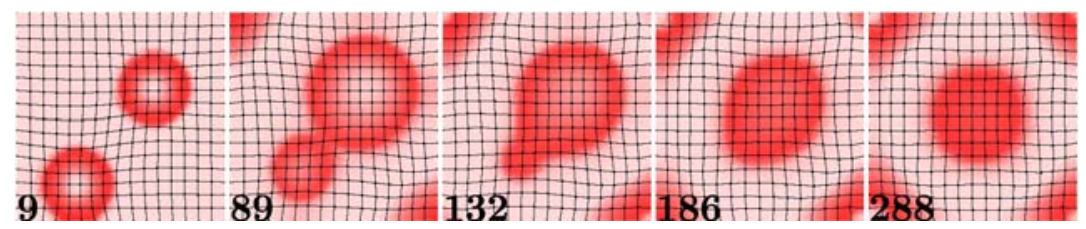

Fig. 7. Drift of two pacemakers starting from non-symmetric locations. Other parameter values were the same as those for Fig. 6.

pacemakers drifted to the center of the tissue and merged with each other to form one stable single pacemaker ( $\operatorname{see} t=480$ ). Note that the final pattern of excitation was the same as that in Fig. 3.

We have also studied the interaction and drift of two non-symmetrically located pacemakers, which started from the initial conditions as illustrated in Fig. 7 (see $t=9$ ). Due to this non-symmetry, the upper pacemaker depolarized more tissue than the lower pacemaker (see $t=89$ ). In this case, we also observed drift of both pacemakers to the center of the tissue, but this proceeded differently. We observed an initial drift of the 'small' lower pacemaker to the 'large' upper pacemaker, which eventually formed one single pacemaking site on the diagonal away from the center (see $t=132$ ). Then, the single pacemaker drifted to the center of the medium where it stabilized (see $t=288$ ). The final pattern in Fig. 6 is the same as that shown in Fig. 3.

From this we conclude that two coexisting pacemakers do not constitute a stable configuration. They merge to form one stable pacemaker that drifts to the center of the medium.

\section{Pacemaker Activity Resulting from Non-Local Stimulation}

In the previous simulations, we only investigated single or multiple pacemaker sites. We now focus on an initial non-local stimulus. To this end, we studied the excitation patterns that occurred as a result of applying an initial stimulation along the vertical mid-line of the tissue as is shown in Fig. 8. We see that during the course 


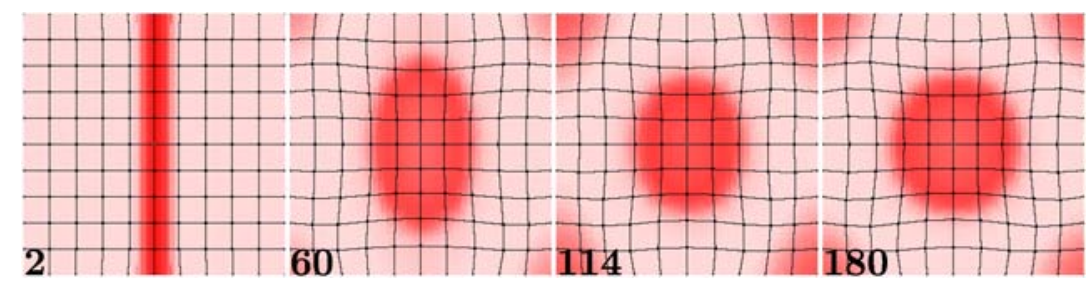

Fig. 8. Pacemaker activity resulting from a non-local stimulation. The size of the medium is $61 \times 61$ grid points. Other parameter values were the same as those for Fig. 3.

of time the oscillating site becomes elliptic (see $t=60$ ), then the asymmetry of the ellipse decreases, and finally we obtained a stable point source that was located in the center of the medium (see $t=180$ ). We also observed that if the initial condition of the line was not located in the center, the line developed to a single point source, which then drifted to the center of the medium, and stabilized (results not shown). Therefore, as for the case of two pacemakers, non-local stimulation also approaches to one stable point pacemaker at the center of the medium.

\section{Pacemaker Drift in a Medium with a Gradient of Excitability}

Pacemaking activity of the heart normally occurs in the sinus node. The sinus node has a complex heterogeneous structure and consists of different cell types, which have different properties with respect to excitability, coupling conductance and oscillation cycle lengths. ${ }^{(39)}$ We studied pacemaker dynamics in a heterogeneous excitable medium with a gradient in excitability induced by changing the parameter $a$ in Eq. (7). The value of $a$ vs $x$ is shown in Fig. 9(left), the lower the value the higher the excitability. Figure 9 (right) shows the dynamics of a single pacemaker
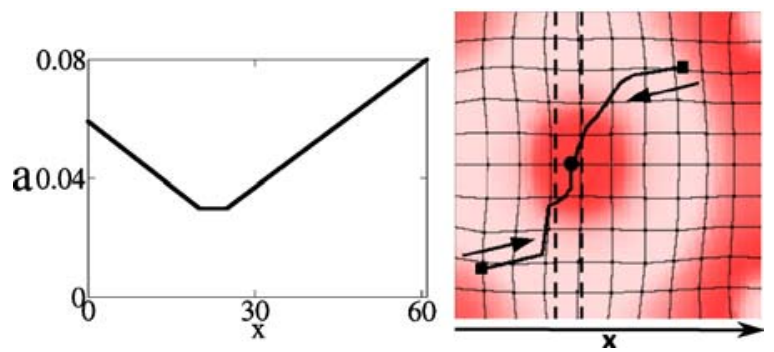

Fig. 9. Pacemaker drift in a heterogeneous medium with respect to the excitability parameter $a$. Left: value of $a$ as function of $x$. Right: Trajectories of drift (solid black lines). The initial starting points are marked with a black square and the end point is marked with black circle. The arrows denote the direction of drift and the dashed lines represent the minimal value of $a$. The grey-scale picture shows the final state of the pacemaker drift. Other parameter values were the same as those for Fig. 8 . 
in such a heterogeneous medium. We studied the dynamics initiated from two different initial conditions (marked by the black squares). The drift trajectories are represented by the black lines, and the directions of the pacemakers are indicated by the black arrows. In both cases, the pacemaker drifted from the initial location to the region located in the middle of the medium in the vertical direction and to the center of the horizontal region where the value of $a$ was minimal. Drift patterns were qualitatively similar to the pattern shown in Fig. 3. For the first few excitations following the initial stimulus, we also observed arc shaped areas of activation, which were subsequently reduced to a point source. For both initial conditions, it took approximately 500 time units to drift to the stable end configuration. We varied the gradient-location in our simulations, and the pacemaker always drifted to the lower value of $a$ (results not shown). The excitability appeared to have a strong influence on the final position of the pacemaker. Note, that for smaller values of $a$, the pacemaker period was shorter. Thus, we observed that pacemakers drifted to regions with shorter period.

\section{DISCUSSION}

In this paper, we studied drift of a single pacemaker and multiple pacemakers in a homogeneous medium. We observed that mechanical deformation has a pronounced effect on drift of pacemakers and induces different excitation patterns. Independent of the initial conditions, multiple pacemakers merged with each other to form one stable pacemaker at the center of the medium. Single pacemakers in a homogeneous medium always drifted to the center of the medium, where they stabilized. Thus, in a homogeneous model the center of the medium is a single global attractor for pacemaking activity. Note that in ref. 29 the attractors for a smaller medium are located differently. We did not study this in this paper, but it would be interesting to investigate this in more detail. Also, here we have only considered pacemaker drift in square/rectangular domains. It would be interesting to study these effects in domains with different shapes and different parametric gradients.

In the presence of a gradient of excitability, we found that pacemaker-drift no longer stabilized at the center, but drifted to the region with the lowest period (as determined by the excitability parameter $a$ ).

Although we use very general descriptions of the medium's excitationmechanics properties and the dynamics of stretch-activated channels, we propose that their effects may be important in cardiac tissue. Indeed, as shown in detailed biophysical models of cardiac tissue, ${ }^{(35)}$ and in experimental studies, ${ }^{(18)}$ stretchactivated channels can depolarize cardiac tissue in a manner similar to that in our computations. The induction of a pacemaker depends on the relation of the depolarizing effect of $I_{S}$ with the excitation properties of cardiac cells. These properties differ substantially throughout the heart, ${ }^{(18)}$ and many types of cardiac cells show 
self-oscillating behavior, even in the absence of applied stretch. Therefore, given the wide variety of properties of cardiac cells and the depolarizing action of the stretch-activated channels in the heart, we propose that the effects of deformation on pacemaking activity can exist for some types of cardiac cells, particularly those that exhibit or are close to self-oscillation dynamics.

Recently, it was shown that calcium overload can lead to pacemaker activity in neonatal rat ventricular myocytes in the form of delayed after depolarizations and that in some cases pacemaker activity drifted throughout the medium. ${ }^{(4)}$ However, the exact cause of the observed pacemaker activity drift is unresolved, and it remains to be seen whether this is a mechanical or electrophysiological effect.

One of the limitations in this study is that we neglected the anisotropic behavior of cardiac tissue, which is important for both the RD and mechanics systems. We chose not to consider these effects, because the main aim of this study was to investigate the basic effects of deformation on pacemaker dynamics.

The onset of pacemaker activity here is probably due to some type of supercritical Hopf bifurcation. Therefore it is reasonable to assume, that close to that bifurcation point the system is sensitive to small spatial and temporal variations and it would be interesting to study that using methods of statistical physics. Note also, that stochastic effects can be also important even for normal wave propagation in the heart as indicated in ref. 32. The influence of these features will likely add additional effects and will be addressed in future studies.

\section{ACKNOWLEDGMENTS}

We are grateful to Prof. P.J. Hunter and Dr. P. Kohl for valuable discussions. This research was funded by the Netherlands Organization for Scientific Research (NWO grant number 814.02.014). M. P. Nash is supported by the Marsden Fund Council from New Zealand Government funding, administered by the Royal Society of New Zealand.

\section{REFERENCES}

1. R. R. Aliev and A. V. Panfilov, A simple two-variable model of cardiac excitation. Chaos, Solitons Fractals 7:293-301 (1996).

2. M. A. Allessie, F. I. M. Bonke, and F. J. G. Schopman, Circus movement in rabbit atrial muscle as a mechanism of tachycardia. Circ. Res. 33:54-62 (1973).

3. G. W. Beeler and H. J. Reuter, Reconstruction of the action potential of ventricular myocardial fibers. J. Physiol. 268:177-210 (1977).

4. M. G. Chang, L. Tung, R. Sekar, J. Cysyk, Y. Qi, L. Xu, E. Marban, and R. Abraham, Calcium overload induces tachyarrhythmias in a $2 \mathrm{D}$ ventricular myocyte experimental model. Heart Rhythm 3(5):S109-S110 (2006).

5. M. Courtemanche, R. Ramirez, and S. Nattel, Ionic mechanisms underlying human atrial action potential properties: insights from a mathematical model. Am. J. Physiol. 275:H301-H321 (1998). 
6. J. M. Davidenko, A. M. Pertsov, R. Salomonsz, W. Baxter, and J. Jalife, Stationary and drifting spiral waves of excitation in isolated cardiac muscle. Nature 355:349-351 (1991).

7. F. Fenton, E. Cherry, H. Hastings, and S. Evans, Multiple mechanisms of spiral wave breakup in a model of cardiac electrical activity. Chaos 12:852-892 (2002).

8. F. Fenton and A. Karma, Vortex dynamics in three-dimensional continuous myocardium with fiber rotation: filament instability and fibrillation. Chaos 8:20-47 (1998).

9. R. FitzHugh, Impulses and physiological states in theoretical models of nerve membrane. Biophys. J. 1:445-465 (1961).

10. M. R. Franz, R. Cima, D. Wang, D. Profitt, and R. Kurz, Electrophysiological effects of myocardial stretch and mechanical determinants of stretch-activated arryhthmias. Circulation 86:968-978 (1992).

11. G. Gerish, Standienpezifische aggregationsmuster bei dictyostelium discoideum. Wihelm. Roux. Arch. Entwick. Org. 156:127-144 (1965)

12. N. A. Gorelova and J. J. Bures, Spiral waves of spreading depression in the isolated chicken retina. J. Neurobiol. 14:353-363 (1983).

13. R. A. Gray and J. Jalife, Ventricular fibrillation and atrial fibrillation are two different beats. Chaos 8:65-78 (1997).

14. A. Hodgkin and A. Huxley, A quantitative description of membrane current and its application to conduction and excitation in nerve. J. Physiol. 117:500-544 (1952).

15. P. Hunter, A. McCulloch, and H. ter Keurs, Modelling the mechanical properties of cardiac muscle. Prog. Biophys. Molec. Biol. 69:289-331 (1998).

16. P. J. Hunter, M. P. Nash, and G. B. Sands, Computational electromechanics of the heart. In: A.V. Panfilov and A.V. Holden (Eds.), Computational Biology of the Heart, pp. 345-407. Wiley, Chichester (1997).

17. R. Imbihl and G. Ertl, Oscillatory kinetics in heterogeneous catalysis. Chem. Rev. 95:697-733 (1995).

18. P. Kohl, P. J. Hunter and D. Noble, Stretch-induced changes in heart rate and rhythm: Clinical observations, experiments and mathematical models. Prog. Biophys. Molec. Biol. 71:91-138 (1999).

19. J. Lechleiter, S. Girard, E. Peralta, and D. Clapham, Spiral calcium wave propagation and annihilation in xenopus laevis oocytes. Science 252:123-126 (1991).

20. W. Li, P. Kohl, and N. Trayanova, Induction of ventricular arrhythmias following mechanical impact: a simulation study in 3d. J. Mol. Histol. 35(7):679-6 (2004).

21. C. Luo and Y. Rudy, A model of the ventricular cardiac action potential. Depolarization, repolarization, and their interaction. Circ. Res. 68:1501-1526 (1991).

22. L. E. Malvern, Introduction to the Mechanics of a Continuous Medium. Prentice-Hall, Inc., Englewood Cliffs, New Jersey (1969).

23. A. D. McCulloch, B. H. Smaill, and P. J. Hunter, Left ventricular epicardial deformation in the isolated arrested dog heart. Am. J. Physiol. 252:H233-H241 (1987).

24. A. P. Muñuzuri, C. Innocenti, J. Flesselles, J. Gilli, K. I. Agladze, and V. I. Krinsky, Elastic excitable medium. Phys. Rev. E 50:R667-R670 (1994).

25. J. Murray, Mathematical Biology. Springer (2002).

26. M.P. Nash, A. Mourad, R. H. Clayton, P. M. Sutton, C. P. Bradley, M. Hayward, D. J. Paterson, and $\mathrm{P}$. Taggart, Evidence for multiple mechanisms in human ventricular fibrillation. Circulation 114(6):530-542 (2006).

27. M. P. Nash and A. V. Panfilov, Electromechanical model of excitable tissue to study reentrant cardiac arrhythmias. Prog. Biophys. Mol. Biol. 85:501-522 (2004).

28. D. Noble, A. Varghese, P. Kohl, and P. Noble, Improved guinea-pig ventricular model incorporating diadic space, $i_{k r}$ and $i_{k s}$, length and tension-dependent processes. Can. J. Cardiol. 14:123-134 (1998) 
29. A. V. Panfilov, R. H. Keldermann, and M. P. Nash, Self-organized pacemakers in a coupled reaction-diffusion-mechanics system. Phys. Rev. Lett. 95(25):258104 (2005).

30. R. Pool, Heart like a wheel. Science 247:1294-1295 (1990).

31. W. Sigurdson, A. Ruknudin, and F. Sachs, Calcium imaging of mechanically induced fluxes in tissue-cultured chick heart: Role of stretch-activated ion channels. Am. J. Physiol. 262:H1110H1115 (1992).

32. M. Spach and J. Heidlage, The stochastic nature of cardiac propagation at a microscopic level. Electrical description of myocardial architecture and its application to conduction. Circ. Res. 76(3):366-380 (1995).

33. K. Ten Tusscher and A. Panfilov, Influence of nonexcitable cells on spiral breakup in twodimensional and three-dimensional excitable media. Phys. Rev. E 68:062902 (2003).

34. K. H. W. J. Ten Tusscher, D. Noble, P. J. Noble, and A. V. Panfilov, A model for human ventricular tissue. Am. J. Physiol. Heart Circ. Physiol. 286:H1573-H1589 (2004).

35. N. Trayanova, W. Li, J. Eason, and P. Kohl, Effect of stretch activated channels on defibrillation efficacy. Heart Rhythm 1:67-77 (2004).

36. C. Weijer, Dictyostelium morphogenesis. Curr. Opin. Genet. Dev. 14:392-398 (2004).

37. R. Yoshida, T. Takahashi, T. Yamaguchi, and H. Ichijo, Self-oscillating gel. J. Am. Chem. Soc. 118:5134-5135 (1996).

38. A. N. Zaikin and A. M. Zhabotinsky, Concentration wave propagation in two-dimensional liquidphase self-organising system. Nature 225:535-537 (1970).

39. H. Zhang, A. Holden, I. Kodama, H. Honjo, M. Lei, T. Varghese, and M. Boyett, Mathematical models of action potentials in the periphery and center of the rabbit sinoatrial node. Am. J. Physiol. Heart Circ. Physiol. 279(1):H397-421 (2000).

40. Z. Zheng, J. Croft, W. Giles, and G. Mensah, Sudden cardiac death in the United States, 1989 to 1998. Circulation 104:2158-2163 (2001). 